\title{
Advances and Novel Approaches in Discrete Optimization
}

\author{
Frank Werner \\ Fakultät für Mathematik, Otto-von-Guericke-Universität Magdeburg, PSF 4120, 39016 Magdeburg, Germany; \\ frank.werner@ovgu.de
}

Received: 17 August 2020; Accepted: 18 August 2020; Published: 25 August 2020

Discrete optimization is an important area of applied mathematics which is at the intersection of several disciplines and covers both theoretical and practical aspects. In the Call for Papers for this issue, I asked for submissions presenting new theoretical results, structural investigations, new models, and algorithmic approaches, as well as new applications of discrete optimization problems. Among the possible subjects, integer programming, combinatorial optimization, optimization problems on graphs and networks, scheduling, logistics, and transportation were mentioned, to name a few.

In response to the Call for Papers, 43 submissions have been received. All submissions have been reviewed, as a rule, by at least three experts in the discrete optimization area. Finally, 17 papers have been accepted for this Special Issue, all of which are of high quality, reflecting the great interest in the area of discrete optimization. This corresponds to an acceptance rate of $39.5 \%$. The authors of these publications come from 13 different countries-China, Pakistan, India, Nepal, Germany, Mexico, USA, Australia, Slovakia, Russia, Korea, Ukraine, and Belarus.

This Special Issue contains both theoretical works and practical applications in the field of discrete optimization. Although many different aspects of discrete optimization have been addressed by the submissions, among the accepted papers there is a major part dealing with scheduling problems as well as graphs and networks. We hope that researchers and practitioners will find many inspirations for future work in the exciting area of discrete optimization. Next, all published papers in this Special Issue are briefly surveyed in increasing order of their publication dates.

The first two papers deal with topological indices of graphs. In the first accepted paper, Ali et al. [1] consider degree-based topological indices and some derived graphs. The goal of this paper is to investigate the chemical behavior of these graphs by means of the topological indices. In particular, the authors find the exact results for the Forgotten index, the Balaban index, reclassified the Zagreb indices, the $A B C_{4}$ index and the $G A_{5}$ index of Hex-derived networks of type 3.

Yang et al. [2] consider the extended adjacency index of a molecular graph. In particular, the authors show some graph transformations which increase or decrease this index. Then they derive the extremal acyclic, unicyclic and bicyclic graphs with a minimum and a maximum extended adjacency index, respectively.

The next three papers deal with particular scheduling problems. Zuo et al. [3] consider two single-machine scheduling problems with possible job rejection and a non-availability interval of the operator simultaneously. The objective is to minimize the sum of either the makespan or total weighted completion time of the accepted jobs and the total cost for the rejected jobs. The authors suggest a pseudo-polynomial solution algorithm as well as a fully polynomial-time approximation scheme.

Wei et al. [4] deal with a problem of transportation and batching scheduling. A single vehicle is considered, and the goal is to minimize total weighted completion time. The main results of this paper are the proof that the problem is NP-hard in the strong sense for any batch capacity of at least 3 as well as a polynomial-time 3-approximation algorithm for the case of a batch capacity of at least 2 .

Li et al. [5] consider a bi-criteria online scheduling problem on parallel batch machines. The batch capacity is unbounded, the processing times of all jobs and batches are equal to one, and the 
objective is to minimize the maximum machine cost subject to a minimum makespan. The authors consider two types of cost functions and present two best possible online algorithms for the problem under consideration.

Pyakurel et al. [6] present efficient algorithms to solve dynamic flow problems with constant attributes as well as generalized problems with partial contraflow reconfiguration in the context of evacuation planning. In particular, a strongly polynomial time algorithm for calculating an approximate solution of the quickest partial contraflow problem on two terminal networks is derived. Numerical results are given for the road network of Kathmandu (Nepal) as the evacuation network.

The next two papers deal again with scheduling problems. Vakhania [7] investigates a single machine scheduling problem with given release dates, due dates, and divisible processing times. The objective is to minimize maximum lateness. He suggests a general method which also leads to useful structural properties of this problem and helps to identify polynomially solvable cases. In particular, for the case of mutually divisible job processing times a polynomial-time algorithm results, and this case turns out to be maximal polynomially solvable one of this problem with non-arbitrary processing times.

Li et al. [8] consider an online scheduling problem with parallel batch machines and linearly deteriorating jobs. The batch capacity is unbounded, and the objective is to minimize the makespan. For the special case of $m=1$, a best possible online algorithm with a competitive ratio of $\left(1+\alpha_{\max }\right)^{f}$ is given, where $f$ denotes the number of job families and $\alpha_{\max }$ gives the maximal deterioration rate of a job. Furthermore, for $m=f \geq 1$, a best possible online algorithm with a competitive ratio of $1+\alpha_{\max }$ is also derived.

$\mathrm{Gu}$ and Yang [9] deal with the max-cut problem. They develop a unique method combining a pointer network and two deep learning strategies, namely supervised learning and reinforcement learning. The pointer network model includes a Long Short-Term Memory network and an Encoder-Decoder. It turned out that their model can be used to solve large-scale max-cut problems heuristically, where for high-dimensional cases, reinforcement learning turned out to be superior to supervised learning.

Cordona-Valdes et al. [10] deal with the multi-product, multi-period capacitated lot sizing problem. Determining the optimal lot size allows shortages resulting in a penalty cost. Two mixed-integer formulations are developed: one model allows shortages, and the other one enforces the fulfillment of the demand. The developed models have been applied to a Mexican fashion retail company within a case study. Both formulations significantly reduced the final inventory costs.

Stas [11] investigates a graph-theoretic subject, namely the crossing number of a graph which is the minimum number of edge crossings over all drawings of the graph in the plane. In particular, he presents this number for the join product $K_{2,3}+C_{n}$, where $K_{2,3}$ is the complete bipartite graph and $C_{n}$ is a cycle on $n$ vertices. The methods applied by the author use several combinatorial properties on cyclic permutations.

Shablya et al. [12] look for new combinatorial generation algorithms. They give basic general methods and investigate one of them based on AND/OR trees. They apply the method of compositae from the theory of generating functions. To show the effectiveness of the suggested modifications, they also derive new ranking and unranking algorithms for several combinatorial sets.

Arora et al. [13] deal with an optimized analysis and planning for power generation and management. They describe several optimization methodologies. In particular, binary variations of the moth flame optimizer and the Harris hawks optimizer are analyzed and tested on 23 benchmark functions, for example, unimodal, multi-modal ones and functions with fixed dimension. The comparison and simulation results demonstrate that their implemented algorithm delivered better results towards the load frequency control problem of a smart grid arrangement compared to earlier methods. 
Pankratov et al. [14] deal with packing problems of irregular 3D objects. By using the phi-function technique, the problem is reduced to the solution of a nonlinear programming model and solved by a multi-start strategy with finding local extreme points. The algorithm has been tested on some benchmark instances.

Lazarev et al. [15] consider the single-machine problem with given release dates and the objective to minimize the maximum job penalty. While this problem is NP-hard in the strong sense, they introduce a dual and an inverse problem, which can be both polynomially solved. The optimal function value of the dual problem is incorporated as a lower bound into a branch and bound algorithm for the original problem. The authors present computational results with this enumerative algorithm for hard benchmark instances with up to 20 jobs. Most of the instances considered can be solved very fast by the proposed algorithm.

Drahos et al. [16] present a method for a conversion between the Logarithmic Number System (LNS) and floating point (FLP) representations using Reduced Instruction Set Computing (RISC). After giving an overview on FLP and LNS number representations, two algorithms of the RISC conversion between both systems using the 'looping in sectors' procedure are presented. The proposed algorithms deliver a very small maximum relative conversion error, and the authors also mention some interesting applications such as camera systems or car control units.

Sotskov et al. [17] consider the two-machine job-shop scheduling problem with interval processing times and makespan minimization. The focus of this paper is how to execute a schedule in the best way. The paper uses the concept of a minimal dominant set of schedules. An online algorithm of the complexity $O\left(n^{2}\right)$ has been developed, where $n$ denotes the number of jobs. Detailed numerical results are given for instances with up to 100 jobs and different maximal percentage errors in the processing times.

Funding: This research received no external funding.

Acknowledgments: As the Guest Editor, I would like to thank all authors for submitting their work to this Special Issue and also all referees for their support by giving timely and insightful reports. My special thanks go to the staff of the journal Mathematics for the good and pleasant cooperation during the preparation of this issue.

Conflicts of Interest: The author declares no conflict of interest.

\section{References}

1. Ali, H.; Binyamin, M.A.; Shafiq, M.K.; Gao, W. On the Degree-Based Topological Indices of Some Derived Networks. Mathematics 2019, 7, 612. [CrossRef]

2. Yang, B.; Manjalapur, M.A.; Sajjan, S.P.; Mathai, M.M.; Liu, J.-B. On Extended Adjacency Index with Respect to Acyclic, Unicyclic and Bicyclic Graphs. Mathematics 2019, 7, 652. [CrossRef]

3. Zuo, L.; Sun, Z.; Lu, L.; Zhang, L. Single-Machine Scheduling with Rejection and an Operator Non-Availability Interval. Mathematics 2019, 7, 668. [CrossRef]

4. Wei, H.; Yuan, J.; Gao, Y. Transportation and Batching Scheduling for Minimizing Total Weighted Completion Time. Mathematics 2019, 7, 819. [CrossRef]

5. Li, W.; Zhai, W.; Chai, X. Online Bi-Criteria Scheduling on Batch Machines with Machine Costs. Mathematics 2019, 7, 960. [CrossRef]

6. Pyakurel, U.; Nath, H.N.; Dempe, S.; Dhamala, T.N. Efficient Dynamic Flow Algorithms for Evacuation Planning Problems with Partial Lane Reversal. Mathematics 2019, 7, 993. [CrossRef]

7. Vakhania, N. Dynamic Restructuring Framework for Scheduling with Release Times and Due-Dates. Mathematics 2019, 7, 1104. [CrossRef]

8. Li, W.; Wang, L.; Chai, X.; Yuan, H. Online Batch Scheduling of Simple Linear Deteriorating Jobs with Incompatible Families. Mathematics 2018, 8, 170. [CrossRef]

9. Gu, S.; Yang, Y. A Deep Learning Algorithm for the Max-Cut Problem Based on Pointer Network Structure with Supervised Learning and Reinforcement Learning Strategies. Mathematics 2020, 8, 298. [CrossRef] 
10. Cardona-Valdes, Y.; Nucamendi-Guillen, S.; Peimbert-Garcia, R.E.; Macedo-Barragan, G.; Diaz-Medina, E. A New Formulation for the Capacitated Lot Sizing Problem with Batch Ordering Allowing Shortages. Mathematics 2020, 8, 878. [CrossRef]

11. Stas, M. Join Products $K_{2,3}+C_{n}$. Mathematics 2020, 8, 925. [CrossRef]

12. Shablya, Y.; Kruchinin, D.; Kruchinin, V. Method for Developing Combinatorial Generation Algorithms Based on AND/OR Trees and Its Application. Mathematics 2020, 8, 962. [CrossRef]

13. Arora, K.; Kumar, A.; Kamboj, V.K.; Prashar, D.; Jha, S.; Shrestha, B.; Joshi, G.P. Optimization Methodologies and Testing on Standard Benchmark Functions of Load Frequency Control for Interconnected Multi Area Power System in Smart Grids. Mathematics 2020, 8, 980. [CrossRef]

14. Pankratov, A.; Romanova, T.; Litvinchev, I. Packing Oblique 3D Objects. Mathematics 2020, 8, 1130. [CrossRef]

15. Lazarev, A.; Pravdivets, N.; Werner, F. On the Dual and Inverse Problems of Scheduling Jobs to Minimize the Maximum Penalty. Mathematics 2020, 8, 1131. [CrossRef]

16. Drahos, P.; Kocur, M.; Haffner, O.; Kucera, E.; Kozakova, A. RISC Conversions for LNS Arithmetic in Embedded Systems. Mathematics 2020, 8, 1208. [CrossRef]

17. Sotskov, Y.N.; Matsveichuk, N.M.; Hatsura, V.D. Schedule Execution for Two-Machine Job-Shop to Minimize Makespan with Uncertain Processing Times. Mathematics 2020, 8, 1314. [CrossRef]

(C) 2020 by the authors. Licensee MDPI, Basel, Switzerland. This article is an open access article distributed under the terms and conditions of the Creative Commons Attribution (CC BY) license (http://creativecommons.org/licenses/by/4.0/). 